\title{
Evaluation of the solid state form of tadalafil in sub-micron thin films using nanomechanical infrared spectroscopy
}

Samaeifar, Fatemeh; Casci Ceccacci, Andrea; Bose-Goswami, Sanjukta; Nielsen, Line Hagner; Afifi, Ahmad; Zor, Kinga; Boisen, Anja

Published in:

International Journal of Pharmaceutics

Link to article, DOI:

10.1016/j.ijpharm.2019.05.010

Publication date:

2019

Document Version

Peer reviewed version

Link back to DTU Orbit

Citation (APA):

Samaeifar, F., Casci Ceccacci, A., Bose-Goswami, S., Nielsen, L. H., Afifi, A., Zor, K., \& Boisen, A. (2019). Evaluation of the solid state form of tadalafil in sub-micron thin films using nanomechanical infrared spectroscopy. International Journal of Pharmaceutics, 565, 227-232.

https://doi.org/10.1016/j.ijpharm.2019.05.010

\section{General rights}

Copyright and moral rights for the publications made accessible in the public portal are retained by the authors and/or other copyright owners and it is a condition of accessing publications that users recognise and abide by the legal requirements associated with these rights.

- Users may download and print one copy of any publication from the public portal for the purpose of private study or research.

- You may not further distribute the material or use it for any profit-making activity or commercial gain

- You may freely distribute the URL identifying the publication in the public portal 


\title{
Evaluation of the solid state form of tadalafil in sub-micron thin films using nanomechanical infrared spectroscopy
}

Fatemeh Samaeifar ${ }^{1,2^{*}}$, Andrea Casci Ceccacci ${ }^{1}$, Sanjukta Bose Goswami ${ }^{1}$, Line Hagner Nielsen ${ }^{1}$, Ahmad Afifi $^{2}$, Kinga Zór ${ }^{1 *}$, Anja Boisen ${ }^{1}$

${ }^{1}$ The Danish National Research Foundation and Villum Foundation's Center for Intelligent Drug Delivery and Sensing Using Microcontainers and Nanomechanics (IDUN), Department of Micro- and Nanotechnology, Technical University of Denmark, Denmark.

${ }^{2}$ Department of Electrical and Computer Engineering, Malek Ashtar University of Technology, Tehran, Iran

Andrea Casci Ceccacci (acc@capres.com), Sanjukta Bose-Goswami (sbos@nanotech.dtu.dk), Line Hagner Nielsen (lihan@nanotech.dtu.dk), Ahmad Afifi (afifi@mut.ac.ir) ,Anja Boisen (anja.boisen@nanotech.dtu.dk)

*Corresponding authors:

Fatemeh Samaeifar, fsamaeifar@yahoo.com, Postal address: No.11, Golestan St., Namaz Sq., Rey, Tehran, Iran. Tel.: +989127751008, Fax: +982122959061

Kinga Zór, kinzo@nanotech.dtu.dk,

Postal address: Technical University of Denmark, Ørsteds Plads, Building 345C, room 101E, 2800 Kgs. Lyngby. Tel.: +45 45255751

\begin{abstract}
Assessing physical stability of drugs is important both in the development as well as in the production phase in the pharmaceutical industry. We used nanomechanical infrared (NAM-IR) spectroscopy based on photothermal response of a nanomechanical resonator, to investigate the solid state forms of tadalafil (TAD), under various storage conditions in sub-micron thin films. The amorphous TAD was stable, when kept at normal storage conditions of $24^{\circ} \mathrm{C}, 45 \%$ relative humidity $(\mathrm{RH})$ and shielded from light, however, it crystallized after four days when it was at stress storage conditions $\left(40^{\circ} \mathrm{C}, 70 \% \mathrm{RH}\right.$, and direct sunlight). Additionally, we found that the signals recorded with NAM-IR were comparable with the attenuated total reflectance Fourier transform infrared spectroscopy (ATR-FTIR) and that NAM-IR proved to be a suitable and time efficient method when evaluating TAD in sub $500 \mathrm{~nm}$ layers.
\end{abstract}

Keywords: Amorphous; Crystalline; NAM-IR; Resonator; Tadalafil 


\section{Introduction}

Oral delivery is the preferred route for drug administration, due to its ease of administration, high patient compliance and cost effectiveness (Aulton and Taylor, 2017). Many drugs in the pipeline from the pharmaceutical industry have a poor aqueous solubility. These drugs cannot dissolve in the available volume of gastrointestinal fluids and thereby, they cannot be absorbed over the intestinal wall and into the blood stream (Lu et al., 2017; Lu et al., 2014).

One strategy to improve the aqueous solubility is to keep the drug in its amorphous form improving the dissolution rate and thus, often the oral bioavailability (Paca et al., 2009; Zhang et al., 2012). However, the amorphous forms are in a thermodynamically unstable state, and therefore over time they tend to spontaneously convert to metastable or stable crystalline forms (Bauer, 2009; Spain, 1995). The onset of crystallization is frequently associated with the molecular mobility of the amorphous matrix (Aso et al., 2004; Yu, 2001), and therefore the physical stability of amorphous drugs can be improved by addition of polymers to create a solid dispersion (Rickert et al., 1984). However, the drug still has a tendency to convert back to a crystalline form (Aso et al., 2004; Yu, 2001), and the conversion can occur during manufacturing, storage and/or dissolution (Nishino et al., 2000). Therefore, among others, the effect of storage conditions such as humidity, temperature and light are important and required to be evaluated (Hancock and Parks, 2000). Various well established techniques are currently used for characterization of the solid state form of drugs such as X-ray powder diffraction (XRPD), differential scanning calorimetry (DSC), Raman spectroscopy, polarized light microscopy (PLM), and infrared (IR) spectroscopy (Byard et al., 2005; Gupta et al., 2003; Mura et al., 2002; Nielsen et al., 2013).

When evaluating layers less than few micrometers the characterization can be challenging (Huth et al., 2012). Conventional DSC requires long analysis times (minutes to hours). In addition, DSC is a destructive method for the samples as in DSC, the glass transition, crystalline and melting peaks are observed by heating the drugs, and the state of drug is detectable using these peaks (Kessler, 2004), therefore unsuitable for the evaluation of the stability of drugs. XPRD method would not be suitable for measuring thin samples since the X-ray beam would measure deeper than the thicknesses of the films (Sarsfield et al., 2006). Conventional IR spectroscopy approaches does not provide high quality results in within a reasonably limited experimental time frame when evaluating sub-micron films (Laroche et al., 2013). Although Raman spectroscopy is a rapid measurement method, nondestructive and requires no sample preparation, it has been indicated that nonaromatic, noncrystalline, hydrophilic excipients have poor Raman scatters compared with drug substances that are normally aromatic heterocycles (Furuyama et al., 2008). With PLM birefringent crystalline material in thin transparent powders can be easily detected. However, the samples must be transparent for this method to work, thus PLM is not suitable for intact tablets (Neilly and Roth, 2016).

For oral administration of drugs, tablets are the most common dosage form. Hence, the drug (e.g. in its amorphous form with or without addition of polymers) needs to be compressed into a tablet (Ramin et al., 2011). Therefore, it is essential to measure crystallinity over time on a tablet, but 
this is challenging with the current methods. The crystallization will often happen on the surface of the tablet and hence, this is important to being able to measure the crystallinity in very thin layers for example in less than $1 \mu \mathrm{m}$ thick layers (Ramin et al., 2011). In addition, when developing controlled sequential drug release surfaces, based on electrostatic layer-by-layer assembly, it is required to perform systematic characterization of layers at the sub-micron level (Laroche et al., 2013). Drugs and polymers are built in separate layers, often with a thickness of 100-200 nm (Moskowitz et al., 2010). Thus, it is of high importance to evaluate the stability and solid state form of the drug as well as the interactions of drug and polymer while building up the layers (Wlodarski et al., 2015).

Nanomechanical infrared spectroscopy (NAM-IR) using filter membrane resonator was recently shown as a sensitive method to performing chemical and morphological measurements on roughly100 pg of sample (Kurek et al., 2017). The principle of NAM-IR is based on the thermal sensitivity of a nanomechanical resonator (Yamada et al., 2013). The photothermal effect yields to a local heat (Bialkowski, 1996) that triggers partial release of native stress in the resonator, causing a resonance frequency drop (Larsen et al., 2013). As the light absorption is wavelength specific, the representation of resonance frequency shift as a function of the incident wavelength becomes a representation of the photothermal spectra. In photothermal spectroscopy the detection of the absorption is decoupled by the light source (Bialkowski, 1996). An advantage of NAM-IR is that only the absorption of the light contributes to the generation of the signal, In this way the shift in the resonance frequency becomes a representation of the absorption spectra and therefore the light that is scattered back does not plays any role in the generation of the photothermal signal (Yamada et al., 2013), so it is free from light-matters based artifacts.

The model drug in this study was tadalafil (TAD), a poorly water soluble drug $(3 \mu \mathrm{g} / \mathrm{ml}$ in aqueous buffer at $\mathrm{pH}$ 7.4) belonging to class II in the Biopharmaceutical Classification System (Wlodarski et al., 2015).

The aim of this paper is investigating the solid state forms of TAD, under various storage conditions in sub-micron thin films using NAM-IR. For this purpose, silicon nitride membrane resonators were used to evaluate crystalline or amorphous TAD in thin films. Spin coating enabled the deposition of 100 to $1100 \mathrm{~nm}$ thick layers of TAD on the membrane in order to create a film which mimics a thin film in the surface of a tablet, as a proof-of-concept. The stability of the amorphous form of TAD, was evaluated with NAM-IR and compared with values obtained with attenuated total reflectance Fourier transform IR (ATR-FTIR). In addition, we studied the solid state form of TAD under various storage conditions.

\section{Material and methods}

\subsection{Membrane fabrication}

Membrane resonators were fabricated based on standard silicon microfabrication techniques(Maluf, 2002) on double-side polished silicon wafers using a single mask process. First, $100 \mathrm{~nm}$ of silicon-rich silicon nitride was deposited on both sides of the silicon substrate by means of low-pressure chemical vapor deposition (LPCVD). Then, a sacrificial layer of silicon 
rich silicon nitride was deposited on the front side of the wafer using plasma enhanced chemical vapor deposition (PECVD) as shown in Fig. 1a. This was followed by patterning the etch masks $(1 \mathrm{~mm} \times 1 \mathrm{~mm})$ on the backside of the wafer by standard photolithography techniques, and the membrane areas were etched away through dry etching (Fig. 1b). Subsequently, the membranes were released by means of $28 \%$ potassium hydroxide (Fig. 1c), and finally, the sacrificial layer was removed by buffered hydrogen fluoride (Fig. 1d).

\subsection{Sample Preparation}

TAD was purchased in the crystalline form (AK Scientific, Inc., Union City, CA, USA) and was dissolved in dimethyl sulfoxide (DMSO). TAD with different concentrations of 5, 10, 15, 20, 39, 60 , and $78 \mathrm{mg} / \mathrm{mL}$ were dissolved in DMSO, and the solid states of the prepared solutions were directly measured using ATR-FTIR. In addition, the states of thin films prepared from these solutions were measured using ATR-FTIR. The results showed that the compounds were amorphous in the concentrations of $5,10,15,20$, and $39 \mathrm{mg} / \mathrm{mL}$ but crystalline at 60 and 78 $\mathrm{mg} / \mathrm{mL}$. Note that the maximum solubility of TAD in DMSO is $78 \mathrm{mg} / \mathrm{mL}$, so this concentration was chosen as a crystalline form of TAD and a half of this (i.e. $39 \mathrm{mg} / \mathrm{mL}$ ) was chosen as amorphous form.

In addition, in order to perform measurements with amorphous TAD as a reference, crystalline powder was melted on a heating plate at $180^{\circ} \mathrm{C}$ for $2 \mathrm{~min}$. The melted TAD was then immediately quench-cooled using liquid nitrogen followed by lightly grinding the amorphous drug with a mortar and pestle.

Solutions of TAD in DMSO were deposited on the fabricated membranes using a spin coater (WS-650M, Laurell Technologies Corporation, USA). In order to overcome the issues of spin coating a low viscosity solution on a hydrophobic silicon nitride membrane, first the surface of the membrane was activated using $\mathrm{O}_{2}$ plasma for $75 \mathrm{~s}$ in order to decrease the surface hydrophobicity. The samples were then heated up to $80^{\circ} \mathrm{C}$ to increase the adhesion strength with the substrate, following spin coating the solution onto the membranes. As a final step, the samples were placed at $50^{\circ} \mathrm{C}$ in DMSO atmosphere for $2 \mathrm{~min}$ in order to achieve a slow solvent evaporation and to obtain uniform films. By controlling the spin coating parameters such as time (s), angular velocity (rpm) and acceleration ( $\mathrm{rpm} / \mathrm{s}$ ), thin films of TAD with different thicknesses between $100 \mathrm{~nm}-1100 \mathrm{~nm}$ were obtained. The thickness and average roughness was measured with a profilometer (Alpha-Step IQ Surface Profiler, KLA-Tencor, USA) (Supporting Information Fig. S1). Experimental results showed that when keeping the time and the acceleration constant at $45 \mathrm{~s}$ and $3600 \mathrm{pm} / \mathrm{s}$ and by changing the speed from 800 to $3000 \mathrm{rpm}$, thicknesses between $100-1100 \mathrm{~nm}$ were obtained for all the samples. The samples were stored at normal conditions $\left(24^{\circ} \mathrm{C}, 45 \% \mathrm{RH}\right.$, and protected from direct sunlight) as well as at stress conditions $\left(40^{\circ} \mathrm{C}, 70 \%\right.$ $\mathrm{RH}$, and exposed to direct sunlight). 


\subsection{NAM-IR Setup}

The NAM-IR setup consisted of a vacuum chamber, parabolic mirror, quantum cascade laser (QCL), laser Doppler vibrometer, and lock-in amplifier as shown in Fig. 2.

The membrane resonator was placed on a piezo ring actuator inside a custom-made aluminum vacuum chamber. The chamber provided electrical feedthrough for piezo actuator, and the chamber had a regular glass window on the top, and zinc selenide $(\mathrm{ZnSe})$ window which is transparent for IR radiation on the bottom. The IR light was emitted by a tunable QCL (LaserTune ${ }^{\circledR}$, Block Engineering, Southborough, MA, USA) and was focused on the back side of the membrane using a parabolic mirror (MPD254254-90-M01, Thorlabs Inc., Newton, NJ, USA). The dynamic behavior of the membrane resonator was read out by a laser Doppler vibrometer (MSA-500, Polytec GmbH, Waldbronn, Germany), and the detuning of the mechanical resonance frequency was monitored and recorded by a lock-in amplifier (HF2LI, Zurich Instruments AG, Zurich, Switzerland).

The reference spectrum of TAD was obtained using a transmission FTIR (Spectrum 100T, PerkinElmer, Waltham, MA, USA), and a ZnSe ATR attachment was used in the same wavelength range as emitted by the IR laser source when evaluating TAD in various thicknesses.

\section{Results and Discussion}

The spectra of TAD in $600 \mathrm{~nm}$ thin film, recorded with NAM-IR, and with ATR-FTIR in both amorphous and crystalline forms, are presented in Fig. 3. The NAM-IR spectra were comparable with the ATR-FTIR reference spectra, for both the crystalline and amorphous forms of TAD in this thickness. The comparability of these two methods has been previously shown by Prashanthi et al., (Prashanthi et al., 2015).

We observed that in the crystalline form, the major peaks measured at 1238, 1262, 1317, 1487, 1501, 1659, and $1679 \mathrm{~cm}-1$ using NAM-IR corresponded closely to the reference ATR-FTIR showing peaks at $1240,1268,1322,1489,1504,1645$, and $1673 \mathrm{~cm}-1$. The last two peaks are attributed to $\mathrm{C}=\mathrm{C}$ aromatic and $\mathrm{C}=\mathrm{O}$ amide (Fig. 4). The double peak of TAD carbonyl groups observed in the range of $1600-1700 \mathrm{~cm}^{-1}$ in the crystalline form was distorted in amorphous form indicating the appearance and participation of both groups in strong hydrogen bonds. Distortion of the carbonyl signal is characteristic of amorphous TAD, as described in the literature (Wlodarski et al., 2016).

Generally, the recommended storage conditions are defined dependent on the drug of interest, however, most commonly the term 'normal storage' refers to temperatures about $25^{\circ} \mathrm{C}$, well ventilated and dry environment (relative humidity level less than 60\%), and protection from intense light (GUIDELINE, 2003; Organization, 2003). The optimum storage condition for TAD is at $25^{\circ} \mathrm{C}$ and it is not recommended to be stored below $15^{\circ} \mathrm{C}$ or above $30^{\circ} \mathrm{C}$ (Food and Administration). In some cases, the storage conditions can change, due to improper placement of the drugs or unforeseen changes in the environment, therefore, it is important to evaluate the stability of the drug at stressed conditions such as increased temperatures, humidity and light exposure. 
When working with various thicknesses of TAD on the membrane resonators using both NAMIR and performing reference measurement with ATR-FTIR, we found that although both NAMIR and ATR-FTIR is rapid and accurate measurement methods in general, NAM-IR was optimum, to evaluate sub $500 \mathrm{~nm}$ films (Supporting Information Table1). For sub $500 \mathrm{~nm}$ films, NAM-IR provided a higher quality spectra relative to the time needed for analysis but ATR spectra were very poor, considering that NAM-IR measurements were achieved in $60 \mathrm{sec}$ for a wavenumber interval of $1,185 \mathrm{~cm}^{-1}$ with $0.5 \mathrm{~cm}$ step size. In the case of ATR-FTIR the acquisition time was approximately $5 \mathrm{~min}$. On the other hand in the range of film thicknesses from $500 \mathrm{~nm}$ to $1100 \mathrm{~nm}$ both NAM-IR and ATR-FTIR measurements were feasible, therefore $600 \mathrm{~nm}$ film thicknesses was used for stability studies in comparison with ATR-FTIR.

The stability of the amorphous form of TAD in $600 \mathrm{~nm}$ thick films on membrane resonators was under four conditions. First, the effect of elevated humidity was studied. The drug was stored at $24^{\circ} \mathrm{C}, 70 \% \mathrm{RH}$, and protected from direct sunlight. Then, the effect of direct light exposure was studied, at $24^{\circ} \mathrm{C}, 45 \% \mathrm{RH}$, while the drug was exposed to direct sunlight. Furthermore, the effect of increased temperature was assessed at $40^{\circ} \mathrm{C}$, while TAD was kept at $45 \% \mathrm{RH}$ and shielded from light. As a control, the amorphous TAD was kept under normal storage conditions at $24^{\circ} \mathrm{C}$, $45 \% \mathrm{RH}$ and protected from light.

We found that the amorphous form of TAD in thin films converted to its crystalline counterpart when exposed to increased humidity and temperature as well as direct sunlight after four days. However, it remained stable when stored under normal storage conditions for up to four days as shown in Fig. 5.and summarized in Table 1. The data obtained with NAM-IR were comparable with the results recorded with the ATR-FTIR used as reference method (Supporting Information Fig. S2).

Additionally, when evaluating the storage conditions of the solid state form of TAD with NAMIR, we found that the film thickness has no effect on the stability, since TAD became crystalline under stressed condition after four days, also in the case of the sub $500 \mathrm{~nm}$ film (Supporting Information S3).

\section{Conclusion}

By studying the solid state form of TAD in spin coated thin films using NAM-IR, we found that after four days the amorphous drug remained stable under normal storage conditions. However, stress conditions $\left(40^{\circ} \mathrm{C}, 70 \% \mathrm{RH}\right.$, and direct sunlight) resulted in crystallization of TAD already after four days. The results obtained with NAM-IR matched with the spectra measured by ATRFTIR in the thicknesses larger than $500 \mathrm{~nm}$. We have shown that NAM-IR enabled fast assessment of drug stability in thin films allowing the recording of high quality spectra of submicron films in less than a minute. Established FTIR based techniques such as ATR-FTIR or Infrared Reflection-Absorption Spectroscopy may provide accurate infrared spectra of thin films, however due to the low sensitivity for sub-micron samples these methods require long acquisition time. In addition, the recorded spectra can be affected by artifacts due to reflections taking place at the sample-substrate interface or to the surface selection rule, which allow to 
probe the vibrations of molecules taking place parallel to the surface (Laroche et al., 2013; Ramin et al., 2011). Instead, NAM-IR provides spectra which are directly generated by only light absorption, free from light-matters based artifacts.

Our findings show the importance of the evaluation of drugs in thin layers, and highlight the advantages of NAM-IR. The presented method is a promising approach for studying drugs in combination with polymers in thin layers when developing layer-by-layer controlled sequential drug release systems as well as in the evaluation of the drug crystallinity on the surface of tablets. 


\section{References}

Aso, Y., Yoshioka, S., Kojima, S., 2004. Molecular mobility-based estimation of the crystallization rates of amorphous nifedipine and phenobarbital in poly (vinylpyrrolidone) solid dispersions. Journal of pharmaceutical sciences 93, 384-391.

Aulton, M.E., Taylor, K.M., 2017. Aulton's Pharmaceutics E-Book: The Design and Manufacture of Medicines. Elsevier Health Sciences.

Bauer, J.F., 2009. Pharmaceutical Solids-The Amorphous Phase. Journal of Validation Technology 15, 63.

Bialkowski, S., 1996. Photothermal spectroscopy methods for chemical analysis. John Wiley \& Sons.

Byard, S.J., Jackson, S.L., Smail, A., Bauer, M., Apperley, D.C., 2005. Studies on the crystallinity of a pharmaceutical development drug substance. Journal of pharmaceutical sciences 94, 1321-1335.

Food, U., Administration, D., CIALIS Highlights of Prescribing Information.

Furuyama, N., Hasegawa, S., Hamaura, T., Yada, S., Nakagami, H., Yonemochi, E., Terada, K., 2008. Evaluation of solid dispersions on a molecular level by the Raman mapping technique. International journal of pharmaceutics 361, 12-18.

GUIDELINE, E., 2003. Stability testing: of existing active substances and related finished products. issued as $\mathrm{CPMP} / \mathrm{QWP} / 122 / 02$.

Gupta, M.K., Vanwert, A., Bogner, R.H., 2003. Formation of physically stable amorphous drugs by milling with Neusilin. Journal of pharmaceutical sciences 92, 536-551.

Hancock, B.C., Parks, M., 2000. What is the true solubility advantage for amorphous pharmaceuticals? Pharmaceutical research 17, 397-404.

Huth, F., Govyadinov, A., Amarie, S., Nuansing, W., Keilmann, F., Hillenbrand, R., 2012. NanoFTIR absorption spectroscopy of molecular fingerprints at $20 \mathrm{~nm}$ spatial resolution. Nano letters 12, 3973-3978.

Kessler, M.R., 2004. Advanced topics in characterization of composites. Trafford Publishing.

Kurek, M., Carnoy, M., Larsen, P.E., Nielsen, L.H., Hansen, O., Rades, T., Schmid, S., Boisen, A., 2017. Nanomechanical Infrared Spectroscopy with Vibrating Filters for Pharmaceutical Analysis. Angewandte Chemie International Edition 56, 3901-3905.

Laroche, G., Fitremann, J., Gherardi, N., 2013. FTIR-ATR spectroscopy in thin film studies: The importance of sampling depth and deposition substrate. Applied Surface Science 273, 632-637.

Larsen, T., Schmid, S., Villanueva, L.G., Boisen, A., 2013. Photothermal analysis of individual nanoparticulate samples using micromechanical resonators. ACS nano 7, 6188-6193.

Lu, W., Dong, X., Qiu, L., Yan, Z., Meng, Z., Xue, M., He, X., Liu, X., 2017. Colorimetric sensor arrays based on pattern recognition for the detection of nitroaromatic molecules. Journal of hazardous materials 326, 130-137.

Lu, W., Li, H., Meng, Z., Liang, X., Xue, M., Wang, Q., Dong, X., 2014. Detection of nitrobenzene compounds in surface water by ion mobility spectrometry coupled with molecularly imprinted polymers. Journal of hazardous materials 280, 588-594.

Maluf, N., 2002. An introduction to microelectromechanical systems engineering. IOP Publishing.

Moskowitz, J.S., Blaisse, M.R., Samuel, R.E., Hsu, H.-P., Harris, M.B., Martin, S.D., Lee, J.C., Spector, M., Hammond, P.T., 2010. The effectiveness of the controlled release of gentamicin from polyelectrolyte multilayers in the treatment of Staphylococcus aureus infection in a rabbit bone model. Biomaterials 31, 6019-6030. 
Mura, P., Faucci, M., Maestrelli, F., Furlanetto, S., Pinzauti, S., 2002. Characterization of physicochemical properties of naproxen systems with amorphous $\beta$-cyclodextrin-epichlorohydrin polymers. Journal of pharmaceutical and biomedical analysis 29, 1015-1024.

Neilly, J., Roth, J., 2016. Detection of Crystalline Components in Amorphous Solid Dispersions by Correlative Imaging Techniques. Microscopy and Microanalysis 22, 1070-1071.

Nielsen, L.H., Gordon, S., Holm, R., Selen, A., Rades, T., Müllertz, A., 2013. Preparation of an amorphous sodium furosemide salt improves solubility and dissolution rate and leads to a faster Tmax after oral dosing to rats. European Journal of Pharmaceutics and Biopharmaceutics 85, 942-951.

Nishino, S.F., Spain, J.C., He, Z., 2000. Strategies for aerobic degradation of nitroaromatic compounds by bacteria: process discovery to field application. Biodegradation of nitroaromatic compounds and explosives 7, 61-65.

Organization, W.H., 2003. Guide to good storage practices for pharmaceuticals. WHO technical report series.

Paca, J., Halecky, M., Barta, J., Bajpai, R., 2009. Aerobic biodegradation of 2, 4-DNT and 2, 6DNT: performance characteristics and biofilm composition changes in continuous packed-bed bioreactors. Journal of hazardous materials 163, 848-854.

Prashanthi, K., Phani, A., Thundat, T., 2015. Photothermal electrical resonance spectroscopy of physisorbed molecules on a nanowire resonator. Nano letters 15, 5658-5663.

Ramin, M.1.A., Le Bourdon, G.n.l., Daugey, N., Bennetau, B., Vellutini, L., Buffeteau, T., 2011. PM-IRRAS investigation of self-assembled monolayers grafted onto $\mathrm{SiO} 2 / \mathrm{Au}$ substrates. Langmuir 27, 6076-6084.

Rickert, D.E., Butterworth, B.E., Popp, J.A., Krahn, D.F., 1984. Dinitrotoluene: acute toxicity, oncogenicity, genotoxicity, and metabolism. CRC critical reviews in toxicology 13, 217-234.

Sarsfield, B., Davidovich, M., Desikan, S., Fakes, M., Futernik, S., Hilden, J., Tan, J., Yin, S., Young, G., Vakkalagadda, B., 2006. Powder X-ray diffraction detection of crystalline phases in amorphous pharmaceuticals. JCPDS-International Centre for Diffraction Data. ISSN, 1097-0002. Spain, J.C., 1995. Biodegradation of nitroaromatic compounds. Annual Reviews in Microbiology 49, 523-555.

Wlodarski, K., Sawicki, W., Haber, K., Knapik, J., Wojnarowska, Z., Paluch, M., Lepek, P., Hawelek, L., Tajber, L., 2015. Physicochemical properties of tadalafil solid dispersions-Impact of polymer on the apparent solubility and dissolution rate of tadalafil. European Journal of Pharmaceutics and Biopharmaceutics 94, 106-115.

Wlodarski, K., Tajber, L., Sawicki, W., 2016. Physicochemical properties of direct compression tablets with spray dried and ball milled solid dispersions of tadalafil in PVP-VA. European Journal of Pharmaceutics and Biopharmaceutics 109, 14-23.

Yamada, S., Schmid, S., Larsen, T., Hansen, O., Boisen, A., 2013. Photothermal infrared spectroscopy of airborne samples with mechanical string resonators. Analytical chemistry 85, 10531-10535.

Yu, L., 2001. Amorphous pharmaceutical solids: preparation, characterization and stabilization. Advanced drug delivery reviews 48, 27-42.

Zhang, M., Li, H., Lang, B., O’Donnell, K., Zhang, H., Wang, Z., Dong, Y., Wu, C., Williams III, R.O., 2012. Formulation and delivery of improved amorphous fenofibrate solid dispersions prepared by thin film freezing. European Journal of Pharmaceutics and Biopharmaceutics 82, 534-544. 


\section{Figures' captions}

Fig. 1. Schematic view of the fabrication process flow. (a) Deposition of silicon-rich silicon nitride with LPCVD and PECVD, respectively, (b) defining the etch mask (c) realizing the membrane using KOH, (d) removing the sacrificial layer.

Fig. 2. Schematic of the experimental setup.

Fig. 3. IR spectra of TAD obtained with NAM-IR and ATR-FTIR. (a) NAM-IR spectroscopy and (b) with ATR-FTIR. The arrows indicate the region where major changes occurred in the amorphous vs. crystalline TAD spectra both in NAM-IR and ATR-FTIR spectra.

Fig 4. Chemical structure of TAD.

Fig. 5. IR spectra of amorphous and crystalline TAD recorded with NAM-IR, observed during four days in various storage conditions. Note that the curves from bottom to top are shifted $0,2,4$, and 6 units, respectively compared with zero reference level. 


\section{Declaration of interests}

$\bigotimes$ The authors declare that they have no known competing financial interests or personal relationships that could have appeared to influence the work reported in this paper.

$\square$ The authors declare the following financial interests/personal relationships which may be considered as potential competing interests:

Sincerely yours,

Fatemeh Samaeifar, $\mathrm{PhD}$

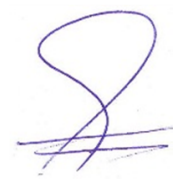


Table 1. Stability of the amorphous form of TAD in thin film in various storage conditions

\begin{tabular}{ccccc}
\hline & Storage conditions & & \multicolumn{2}{c}{ Solid state form } \\
\hline Temperature & $\begin{array}{c}\text { Relative } \\
\text { humidity }\end{array}$ & $\begin{array}{c}\text { Direct } \\
\text { sunlight }\end{array}$ & Day 1 & Day 4 \\
\hline $24^{\circ} \mathrm{C}$ & $70 \%$ & Shielded & Amorphous & Crystalline \\
$24^{\circ} \mathrm{C}$ & $45 \%$ & Exposed & Amorphous & Crystalline \\
$40^{\circ} \mathrm{C}$ & $45 \%$ & Shielded & Amorphous & Crystalline \\
$24^{\circ} \mathrm{C}$ & $45 \%$ & Shielded & Amorphous & Amorphous \\
\hline
\end{tabular}


Table S1. Comparison between NAM-IR and ATR-FTIR in different thicknesses, considering the quality of the spectra relatively to the time needed for analysis

\begin{tabular}{|c|c|c|}
\hline Thickness (nm) & ATR-FTIR & NAM-IR \\
\hline$>1000$ & $\checkmark$ & $x$ \\
\hline $500-1000$ & $\checkmark$ & $\checkmark$ \\
\hline$<500$ & $x$ & $\checkmark$ \\
\hline
\end{tabular}


(a)

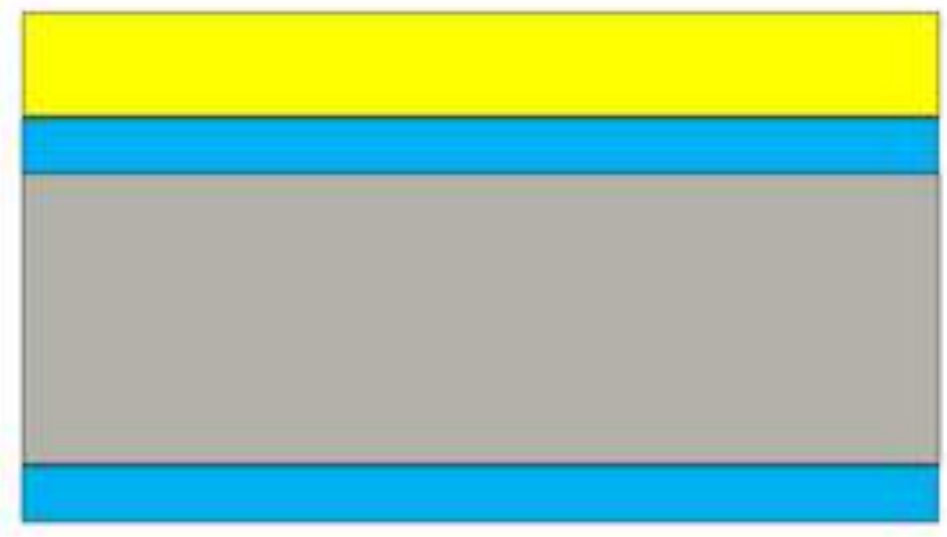

(c)
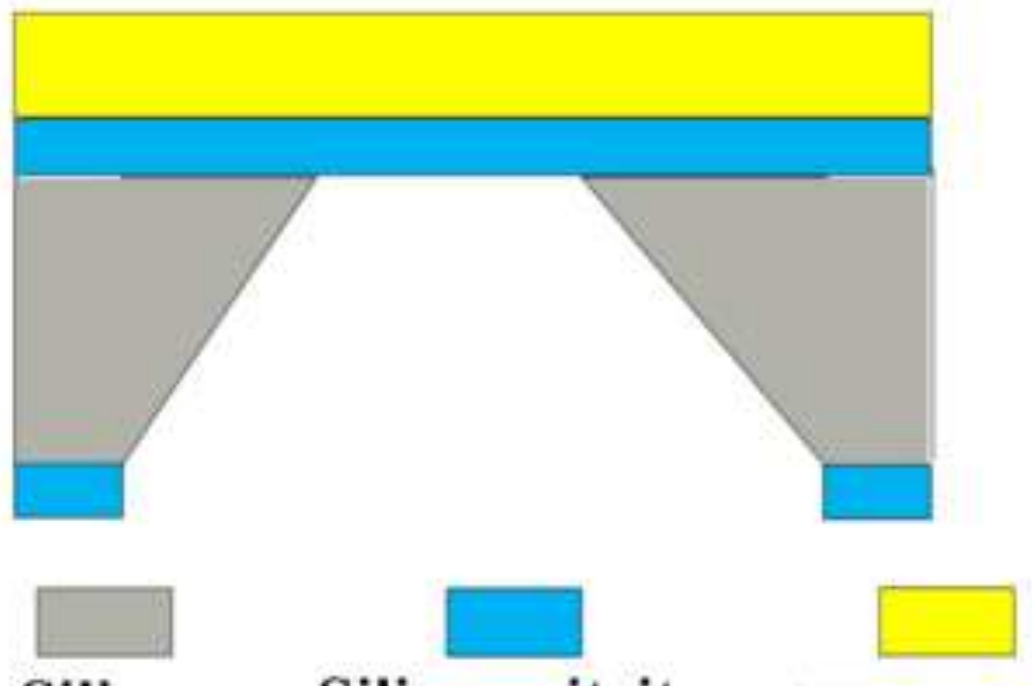

Silicon Silicon nitrite Silicon nitrite (LPCVD) (PECVD) (b)

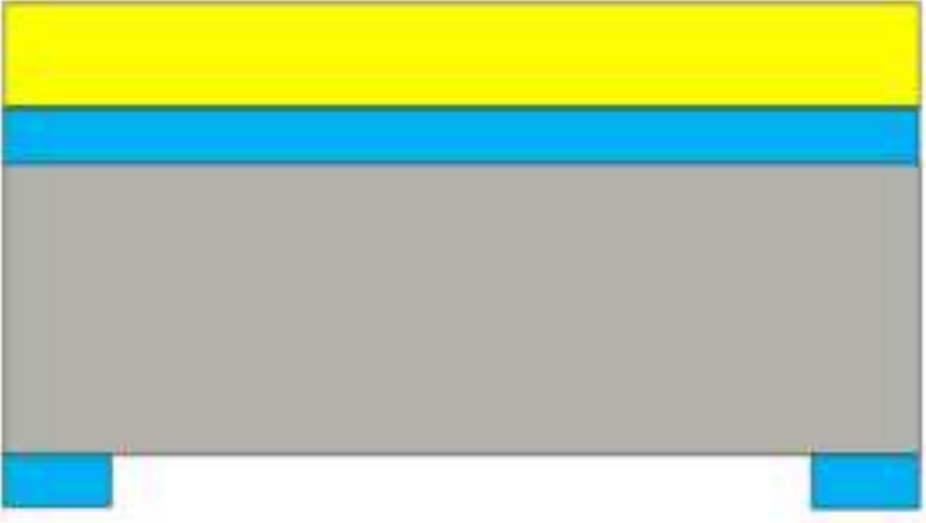

(d)

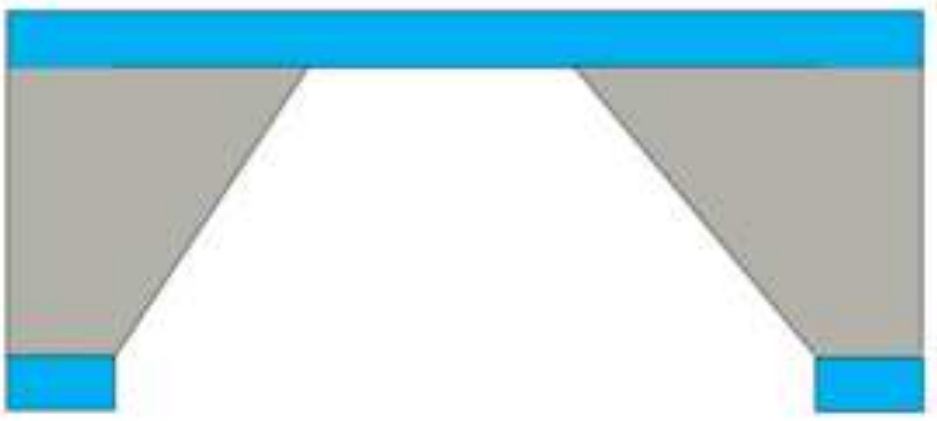



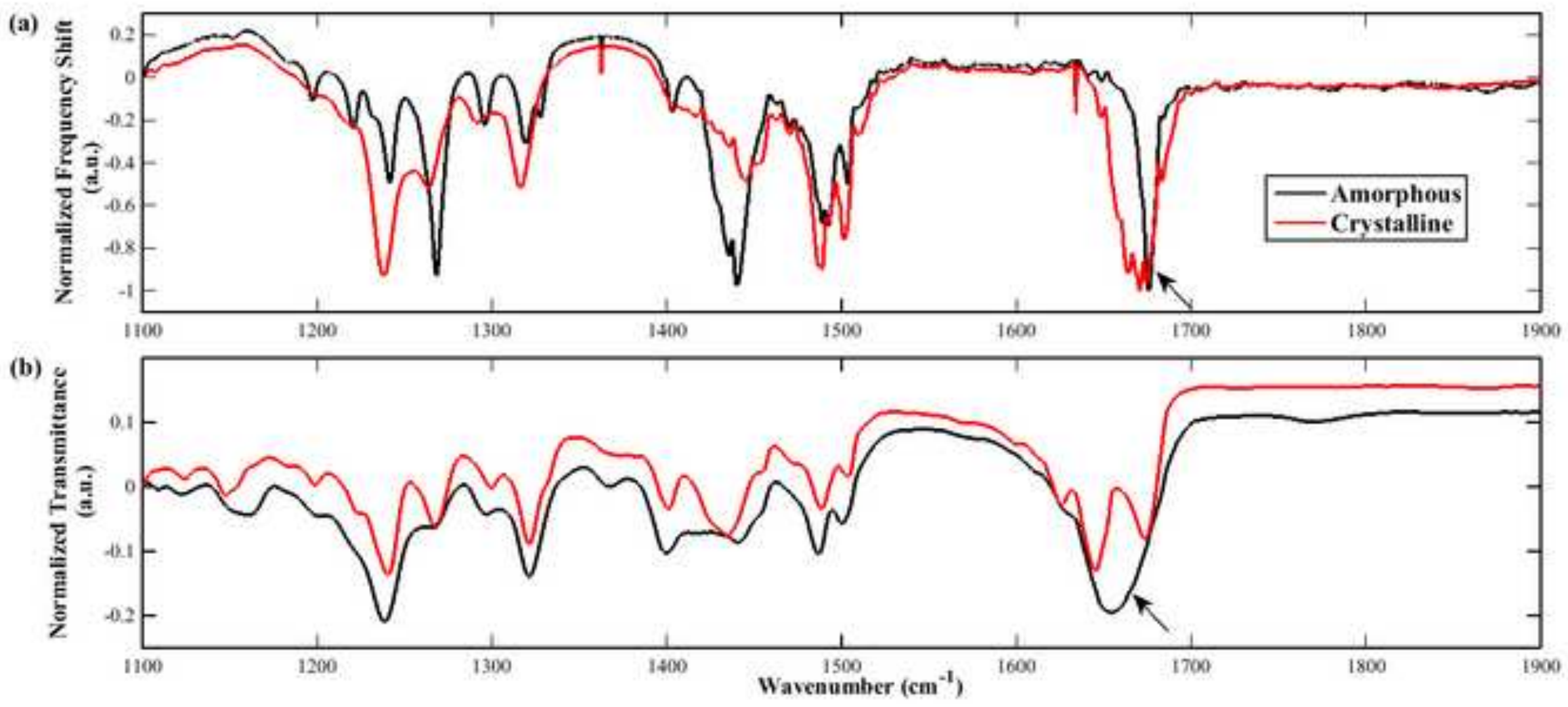


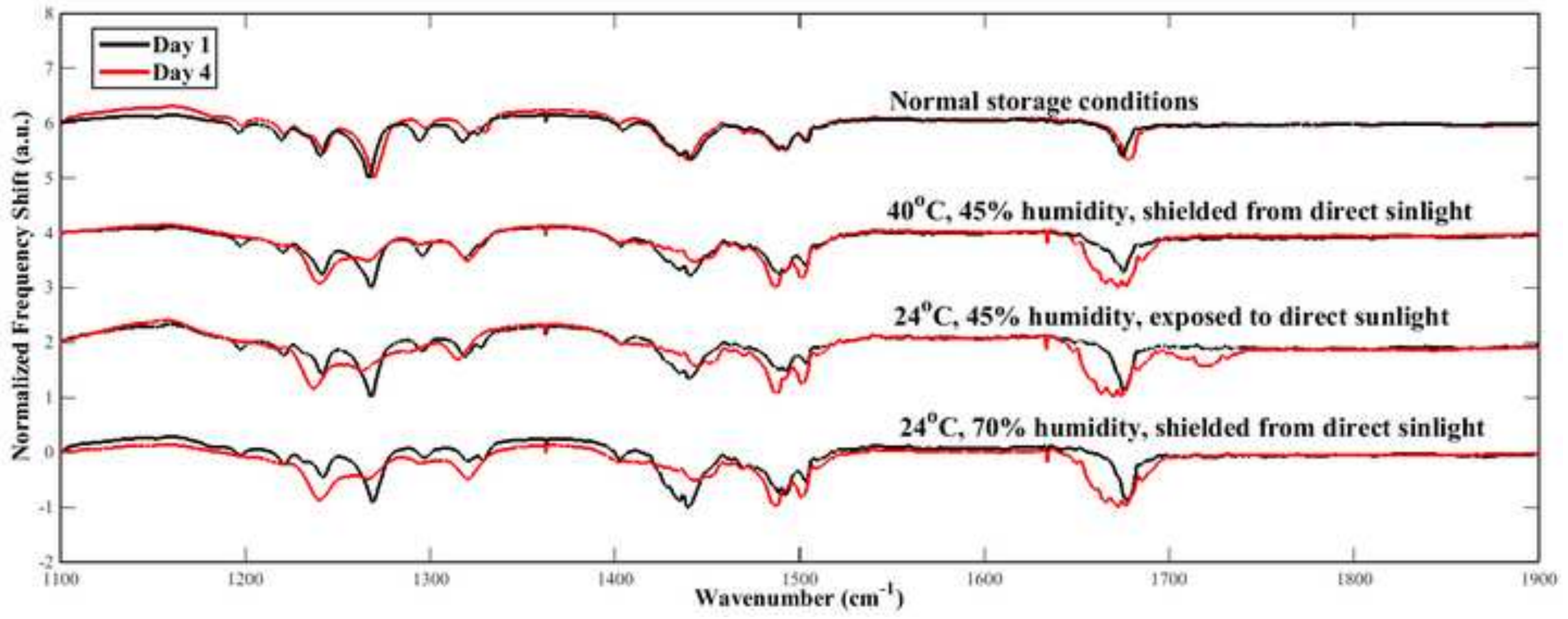



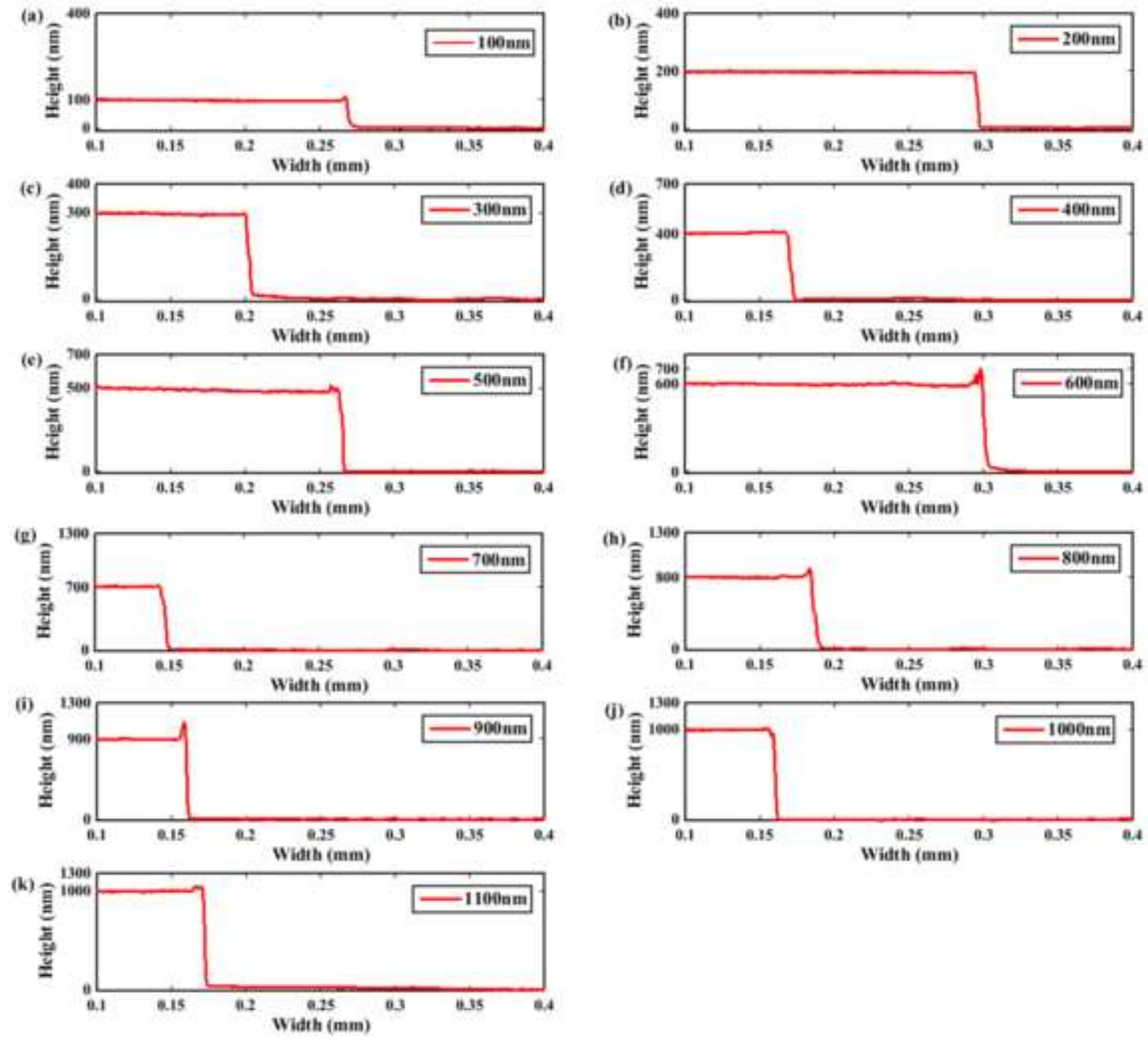


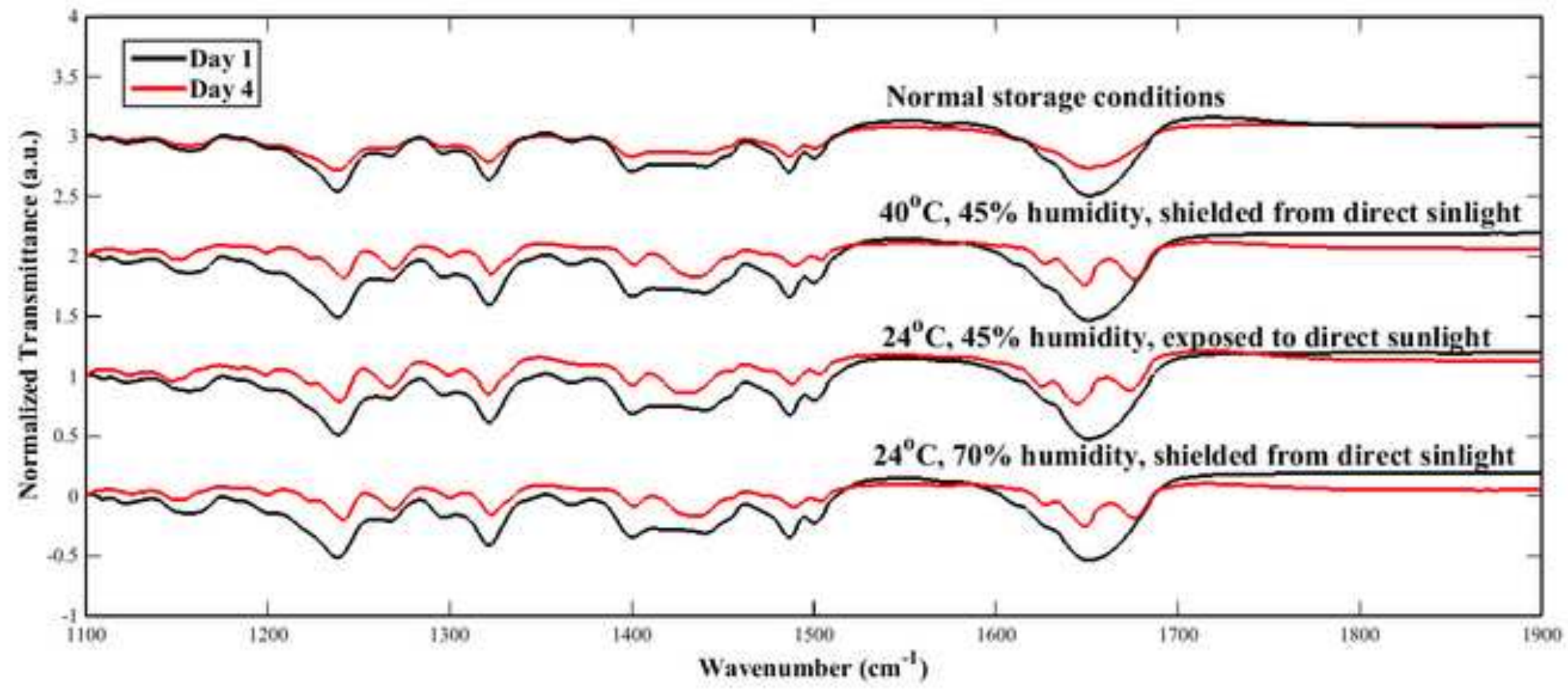




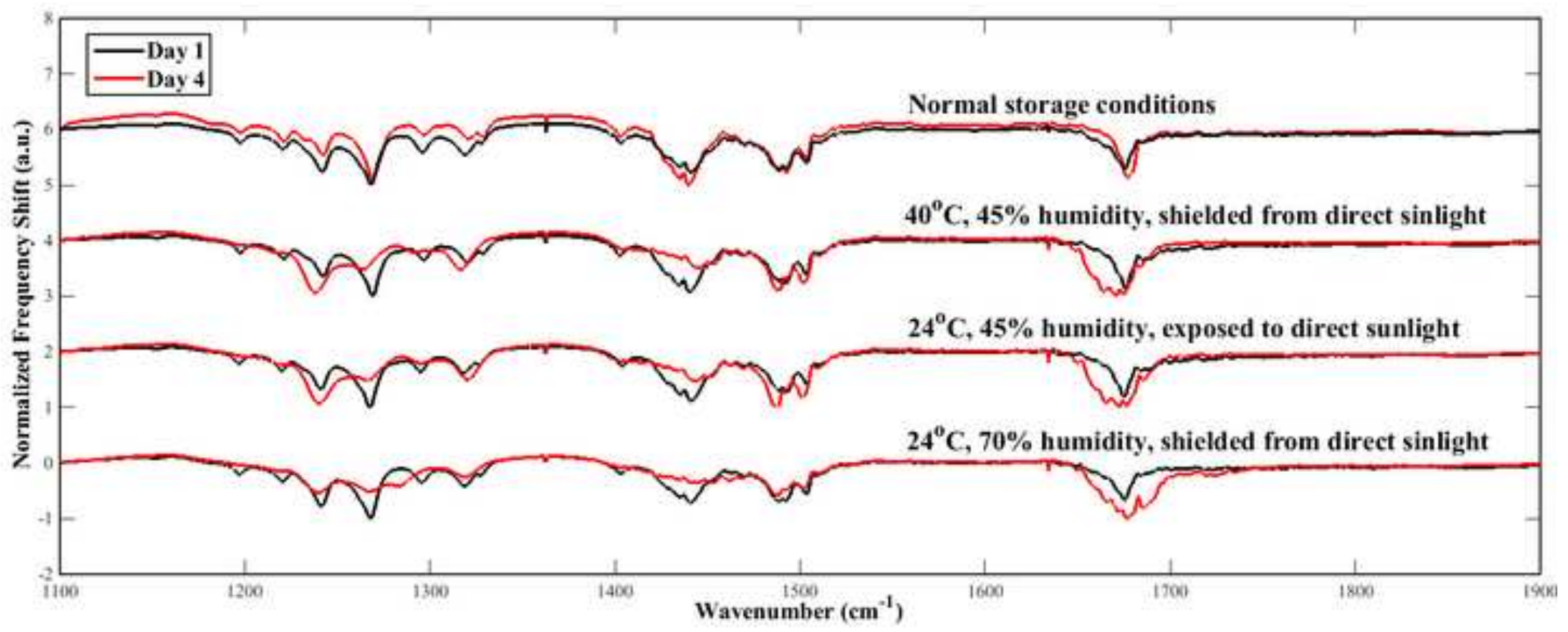


Supplementary Material
Click here to download Supplementary Material: Supporting Information.pdf

Supplementary Material
Click here to download Supplementary Material: Supporting Information.pdf

\begin{abstract}
che here to download Supplementary Material: Supporting Information.pdf
\end{abstract}
Information.pdf

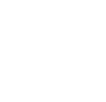

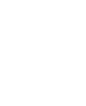

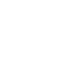

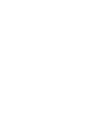

(n) 